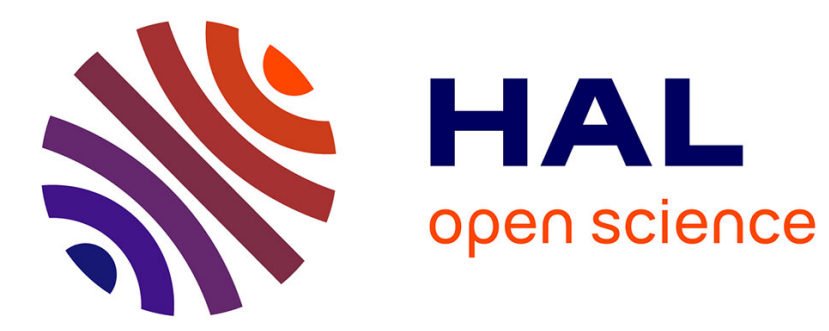

\title{
Investigating the Pressure-Induced Amorphization of Zeolitic Imidazolate Framework ZIF-8: Mechanical Instability Due to Shear Mode Softening
}

Aurélie U. Ortiz, Anne Boutin, Alain H. Fuchs, François-Xavier Coudert

\section{- To cite this version:}

Aurélie U. Ortiz, Anne Boutin, Alain H. Fuchs, François-Xavier Coudert. Investigating the Pressure-Induced Amorphization of Zeolitic Imidazolate Framework ZIF-8: Mechanical Instability Due to Shear Mode Softening. Journal of Physical Chemistry Letters, 2013, 4 (11), pp.1861-1865. $10.1021 / \mathrm{jz} 400880 \mathrm{p}$. hal-02116930

\author{
HAL Id: hal-02116930 \\ https://hal.science/hal-02116930
}

Submitted on 1 May 2019

HAL is a multi-disciplinary open access archive for the deposit and dissemination of scientific research documents, whether they are published or not. The documents may come from teaching and research institutions in France or abroad, or from public or private research centers.
L'archive ouverte pluridisciplinaire HAL, est destinée au dépôt et à la diffusion de documents scientifiques de niveau recherche, publiés ou non, émanant des établissements d'enseignement et de recherche français ou étrangers, des laboratoires publics ou privés. 


\title{
Investigating the Pressure-Induced Amorphization of Zeolitic Imidazolate Framework
}

\section{ZIF-8: Mechanical Instability due to Shear Mode Softening}

Aurélie U. Ortiz, ${ }^{\dagger}$ Anne Boutin, ${ }^{\dagger}$ Alain H. Fuchs,${ }^{\dagger}$ and François-Xavier Coudert ${ }^{*}, \dagger$

$\dagger$ CNRS and Chimie ParisTech, 11 rue Pierre et Marie Curie, 75005 Paris, France

† Département de Chimie, École Normale Supérieure, CNRS-ENS-UPMC, 24 rue Lhomond, 75005

Paris, France

\begin{abstract}
We provide the first molecular dynamics study of the mechanical instability that is the cause of pressure-induced amorphization of zeolitic imidazolate framework ZIF-8. By measuring the elastic constants of ZIF-8 up to the amorphization pressure, we show that the crystal-to-amorphous transition is triggered by the mechanical instability of ZIF-8 under compression, due to shear mode softening of the material. No similar softening was observed under temperature increase, explaining the absence of temperature-induced amorphization in ZIF-8. We also demonstrate the large impact of the presence of adsorbate in the pores on the mechanical stability and compressibility of the framework, increasing its shear stability. This first molecular dynamics study of ZIF mechanical properties under variations of pressure, temperature and pore filling opens the way to a more comprehensive understanding of their mechanical stability, structural transitions and amorphization.
\end{abstract}

Table of Content graphic:

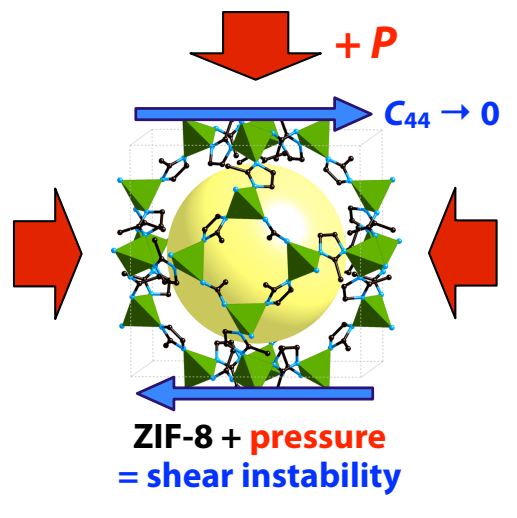

Keywords: metal-organic frameworks, shear instability, mechanical properties, molecular simulation, elastic constants, structural transition, nanoporous materials 
Zeolitic imidazolate frameworks (ZIF) are a subclass of metal-organic frameworks (MOF) $)^{1,2,3,4}$ that feature imidazolate linkers bridging metal centers to form three-dimensional porous crystalline solids isomorphous to zeolitic frameworks. ${ }^{5,6,7,8}$ ZIFs have recently gained considerable attention for their potential applications because they inherit desirable qualities from both worlds: the tunable porosity, structural flexibility and the functionalization of the internal surface of the MOFs, as well as the thermal, mechanical and chemical stability of the zeolites. Moreover, the similarities between the metalimidazolate four-fold coordination chemistry and the corner-sharing $\mathrm{SiO}_{4}$ tetrahedra from which zeolites are built mean that many ZIF topologies can potentially be synthesized. Indeed, over 100 different ZIF

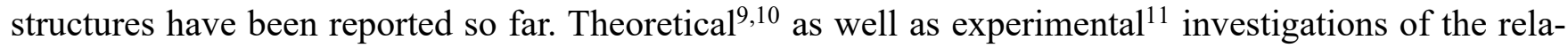
tive stabilities of these structures indicate that they have a relatively low enthalpy of formation, and that many more polymorphs should be accessible under mild synthesis conditions. The ZIF structures reported in the literature so far demonstrate large gamut of attractive structural and physicochemical properties, with great potential for applications in fluid separation, ${ }^{12,13,14,15} \mathrm{CO}_{2}$ capture, ${ }^{16}$ sensing, ${ }^{17}$ encapsulation and controlled delivery. ${ }^{18}$

Given their larger number of potential applications, issues of thermal and mechanical stability of flexible MOFs in general, ${ }^{19,20,21}$ and ZIFs in particular, ${ }^{22}$ are of high importance. A number of recent studies have addressed the question of ZIF stability at high temperature, showing temperature-induced amorphization of some ZIFs (ZIF-1, ZIF-3, ZIF-4), ${ }^{23}$ while others do not amorphize at high temperature (including the prototypical ZIF-8). ${ }^{24}$ On the other hand, all ZIF frameworks studied so far have shown pressure-induced amorphization at modest, industrially accessible pressure. ZIF-8 ${ }^{5}$ (Fig. 1) undergoes irreversible amorphization under nonhydrostatic mechanical compression as low as $0.3 \mathrm{GPa},{ }^{25}$ as well as under ball-milling. ${ }^{26}$ The pressure-induced amorphization of ZIF-4 ${ }^{5}$ (Fig. 1) was also studied: Bennett et al. ${ }^{27}$ showed that amorphization occurs at various values of hydrostatic pressure depending on the presence or absence of solvent molecules in the pores of the material, as well as the nature of the hydrostatic 
medium. The evacuated ZIF-4 demonstrated amorphization at very low pressure $(0.35 \mathrm{GPa})$, while the presence of DMF molecules in its pores could shift amorphization to higher pressure and lead to the existence of an intermediate monoclinic crystalline phase (ZIF-4-I).

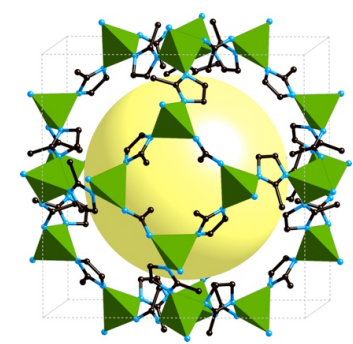

ZIF-8 (SOD)

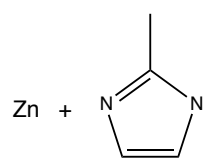

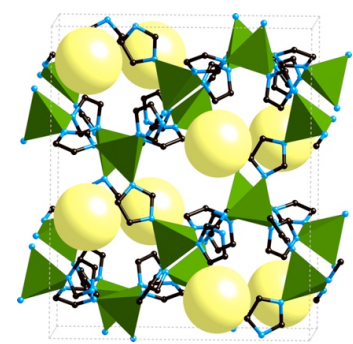

ZIF-4 (cag)

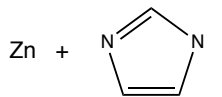

Figure 1. Imidazolate linker, structure and topology of ZIF-4 and ZIF-8. Yellow spheres represent the free volume within the frameworks.

While all the experimental studies of pressure-induced amorphization cited above have taken great care in identifying the structure of the various amorphous ZIF phases, there is a lack of experimental information on the nature of the crystal-to-amorphous transition, the reasons behind its occurrence and its apparent universality under mechanical pressure. This is in good part because the amorphization happens at such low pressure that little data is available on the stressed ZIFs. Furthermore, a recent study by Tan et al. ${ }^{22}$ measured the shear modulus of ZIF-8, which they find to be quite low, at around $1 \mathrm{GPa}$. Tan et al. also suggested that the pressure-induced amorphization of ZIF-8 may be linked to this low shear modulus. However, the pressure-induced amorphization is observed at pressure lower than $1 \mathrm{GPa}$, and in addition a direct link between these two properties has yet to be demonstrated. In this work, we report the evolution of the mechanical properties of ZIF-8, as a function of both external stress and adsorbate loading, in order to elucidate the nature of the pressure-induced crystal-to-amorphous transition. 
Figure 2 presents the evolution of the unit cell parameter and elastic constants of ZIF-8 as a function of mechanical pressure, at $300 \mathrm{~K}$, for an isotropic compression corresponding to hydrostatic experimental conditions with a non-penetrating hydrostatic fluid. Both the average lattice parameters and the elastic constants were obtained from constant-stress $(N, \sigma, T)$ molecular dynamics (MD) simulations by analysis of the fluctuations of unit cell vectors ${ }^{28,29}$ (the so-called strain-fluctuation method), where the elastic stiffness tensor $C_{i j k l}$ is obtained by:

$$
\left(\frac{k_{\mathrm{B}} T}{V}\right) C_{i j k l}^{-1}=\left\langle\epsilon_{i j} \epsilon_{k l}\right\rangle-\left\langle\epsilon_{i j}\right\rangle\left\langle\epsilon_{k l}\right\rangle
$$

with $\varepsilon$ the unit cell strain and $V$ the unit cell volume. We described the fully flexibly ZIF-8 framework using the force field of Zhang et al, which was calibrated to reproduce the ZIF-8 structure, bulk modulus and sorption-induced structural transformation ${ }^{30}$ (see Supporting Information for simulation details).

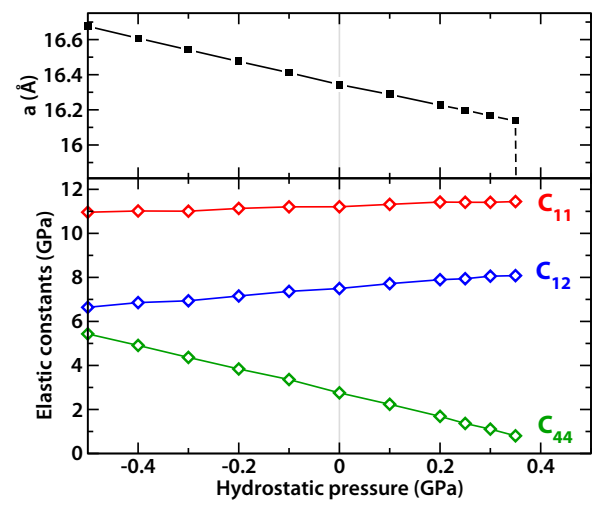

Figure 2. Unit cell parameter $a$ and elastic constants $C_{11}, C_{12}$ and $C_{44}$ of ZIF-8 as a function of hydrostatic pressure at $77 \mathrm{~K}$.

First, direct observation of the MD trajectories shows that the ZIF-8 framework is stable at pressures lower than $0.4 \mathrm{GPa}$, but unstable at higher pressure. In the range of pressures from $-0.5 \mathrm{GPa}$ to $0.35 \mathrm{GPa}$ (where negative pressures indicate tension and positive pressures indicate compression), ZIF-8 shows linear elasticity with a bulk modulus of $E=8 \mathrm{GPa}$, in good agreement with the values determined experimentally (from Brillouin scattering: $7.7 \mathrm{GPa}{ }^{22}$ from pressure-induced lattice variations: 6.5 $\left.\mathrm{GPa}^{25}\right)$. At $P \geq 0.4 \mathrm{GPa}$, however, we observe a rapid collapse of the framework, demonstrating its 
instability under pressure. The value of this critical pressure is, again, in good agreement with the experimental data of Chapman et al. who observed amorphization beyond $0.34 \mathrm{GPa}$ under hydrostatic conditions. $^{25}$

To find the fundamental cause of this instability we look at the evolution of the elastic constants of ZIF-8 as a function of applied pressure (Fig. 2, bottom panel). Because the ZIF-8 framework is cubic, its mechanical properties are fully described by only 3 elastic constants: $C_{11}, C_{12}$ and $C_{44}$, which are respectively the modulus for axial compression, dilation on compression, and shear. First, we see that the values obtained for the unstressed crystal $(P=0)$ using this methodology are in good agreement with the experimental values obtained by Brillouin scattering: the agreement is very good for $C_{11}$ and $C_{12}$ (this work: 11.3 and $7.6 \mathrm{GPa}$; ref. 22: 9.5 and $6.9 \mathrm{GPa})$, and reasonable for $C_{44}(2.7 \mathrm{GPa}$ vs. $0.97 \mathrm{GPa})$ considering how challenging is the accurate prediction of absolute values of elastic constants. More important is the pressure-dependence of the elastic constants, where we can see that $\mathrm{C}_{44}$ diminishes drastically under compression of the crystal, a phenomenon known as shear-mode softening. This decrease leads to elastic instability of the ZIF-8 framework, since the Born stability conditions for a cubic crystal under hydrostatic pressure $P$ can be written as: ${ }^{31}$

$$
C_{11}+2 C_{12}+P \geq 0 ; C_{11}-C_{12} \geq 2 P ; C_{44} \geq P
$$

At $P=0.4 \mathrm{GPa}$ the third condition, which indicates stability under shear deformation, breaks down and the ZIF-8 framework becomes mechanical unstable. We thus conclude that the amorphization of ZIF-8 under pressure is triggered by elastic instability, itself due to a pressure-induced softening of the (already relatively soft) shear deformation mode of the material. While it is the first time such a mechanism is demonstrated for amorphization of a MOF, it is known to be the underlying cause of pressureinduced phase transitions in some dense inorganic solids (e.g. the halite-CsCl-type transition of $\mathrm{MgO}$, or the stishovite- $\mathrm{CaCl}_{2}$-type transition in $\mathrm{SiO}_{2}{ }^{32}$ ) as well as the pressure-induced amorphization of coesite. $^{33}$ 
In their recent study of ZIF-4 amorphization, Bennett et al. ${ }^{27}$ have demonstrated that both the onset of amorphization and the bulk modulus of ZIF-4 depend highly on the presence or absence of solvent molecules in its pores. In particular, they showed that the evacuated framework was much more flexible. Moreover, it was shown in some soft porous crystals, such as the MIL-53 family, that adsorptioninduced stress can lead to structural transitions of large amplitude. ${ }^{34}$ These considerations are of critical importance for practical applications in real conditions. We have thus investigated the elastic constants and mechanical stability of ZIF-8 with methane adsorbed inside its pores. Methane was chosen as a generic guest molecule for this study because of its apolar nature and the absence of specific guest-ZIF interactions, in order to better understand the generic effect of pore volume filling on mechanical properties. Table I reports the evolution of ZIF-8 elastic constants for $\mathrm{CH}_{4}$ loadings between 0 and 18 molecules per unit cell (which corresponds to methane uptake at 100 bar and $300 \mathrm{~K}$; see isotherm in Fig. S1). While the effect of pore filling is modest on the $C_{11}$ and $C_{12}$ elastic constants, it drastically increases the shear modulus of ZIF-8, with a variation of $C_{44}$ from $2.7 \mathrm{GPa}$ in the evacuated material to $4.0 \mathrm{GPa}$ with 18 methane molecules per unit cell. This increased resistance to shear is directly linked to the higher density of the guest-filled material, for which a shear motion implies a larger number of repulsive guest-guest and guest-host close contacts.

Table I. Elastic constants of the ZIF-8 framework at $300 \mathrm{~K}$ for various values $\mathrm{CH}_{4}$ loading, at $300 \mathrm{~K}$ and 0 GPa.

\begin{tabular}{cccc}
\hline $\begin{array}{c}\mathrm{CH}_{4} / \text { unit } \\
\text { cell }\end{array}$ & $\begin{array}{c}C_{11} \\
(\mathrm{GPa})\end{array}$ & $\begin{array}{c}C_{12} \\
(\mathrm{GPa})\end{array}$ & $\begin{array}{c}C_{44} \\
(\mathrm{GPa})\end{array}$ \\
\hline 0 & 11.21 & 7.49 & 2.75 \\
3 & 11.26 & 7.52 & 2.89 \\
6 & 11.29 & 7.55 & 2.96 \\
11 & 11.33 & 7.58 & 3.18 \\
18 & 11.38 & 7.66 & 4.00 \\
\hline
\end{tabular}




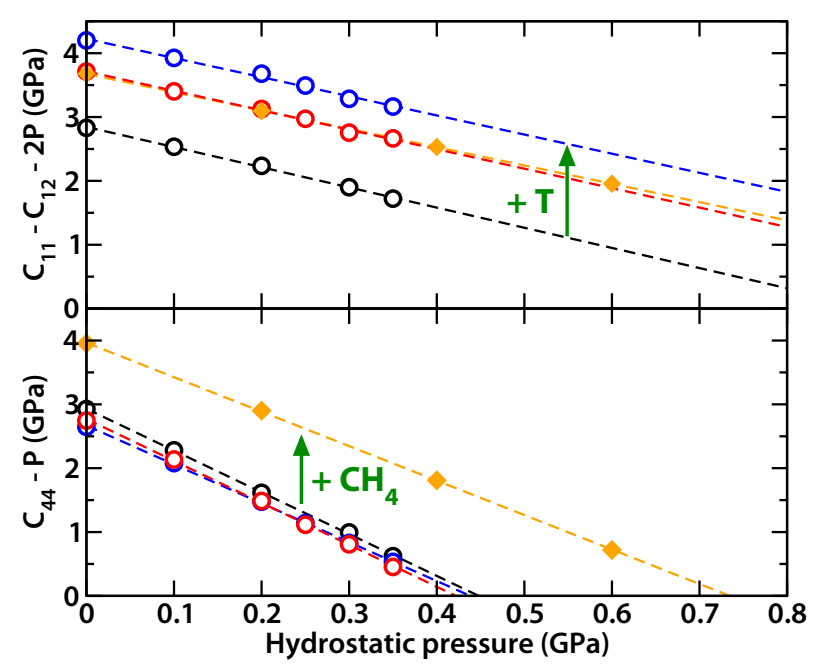

Figure 3. Evolution of the stability criteria for compression (upper panel, $\lambda=C_{11}-C_{12}-2 P$ ) and shear (lower panel, $\mu=C_{44}-P$ ) of ZIF-8 as a function of hydrostatic pressure. Black: empty ZIF-8 at $77 \mathrm{~K}$; red: empty ZIF-8 at $300 \mathrm{~K}$; blue: empty ZIF-8 at $500 \mathrm{~K}$; orange: ZIF-8 with $18 \mathrm{CH}_{4}$ at $300 \mathrm{~K}$.

Moreover, MD simulations of ZIF-8 at this loading and under hydrostatic compression have revealed its increased mechanical stability compared to the empty ZIF-8: the material is stable under compression up to $0.65 \mathrm{GPa}$, while it collapses at higher pressure. The pressure dependence of the elastic constants is shown in Fig. 3 in the form of the stability criteria for compression $\left(\lambda=C_{11}-C_{12}-2 P\right)$ and shear $(\mu=$ $C_{44}-P$ ), both of which must be positive for the system to be mechanically stable. Fig. 3 demonstrates that pressure-induced amorphization in methane-loaded ZIF-8 is, like the evacuated material, triggered by a mechanical instability due to shear softening, but that the onset of amorphization is delayed due to the larger shear modulus of the unstressed material. We also plot on Fig. 3 the evolution of elastic constants at $77 \mathrm{~K}$ and $500 \mathrm{~K}$. No temperature-induced softening is observed: $C_{44}$ is almost unchanged, while $C_{11}$ actually increases. The absence of elastic softening at higher temperature is in good agreement with the fact that no amorphization of ZIF-8 upon heating has been reported in the literature.

Preliminary results on the elastic constants and mechanical stability of the ZIF-4 framework under hydrostatic pressure (Fig. S2) point to the same mechanism for its pressure-induced amorphization. A softening of the shearing modes (three of them because the ZIF-4 framework is orthorhombic) is also found 
to be associated with mechanical instability under compression, though the value of transition pressure obtained by this study does not fall in line with the experimental results and further work needs to be done to refine the force field used for the simulation to account for the differences in coordination bonds and angles between ZIF-8 (for which the force field was optimized) and ZIF-4. Nonetheless, the fact that the very different ZIF-8 and ZIF-4 frameworks both undergo pressure-induced amorphization due to shear instability hints that the phenomenon may be generic. We thus conclude that the porous ZIF phases, though they retain zeolite-like framework topologies, do not possess the mechanical stability of zeolites because of the weaker $\mathrm{Zn}$-imidazolate coordinative bonds, compared to the strong $\mathrm{Si}-\mathrm{O}$ bond. Moreover, their very high porosity gives rise to low resistance to shear and pressure-induced shear softening, which in turn is the cause for their mechanical instability at low pressure.

Finally it should be noted that, while the molecular dynamics simulations reported in this work are helpful to understand how the mechanical instability of ZIFs develop depending on thermodynamic conditions, they unfortunately cannot yield direct information on the nature of the amorphous phase obtained and do not explain why the amorphous phase is preferred to a hypothetical denser crystalline phase. All simulations of ZIF-8 at high pressure, in which the framework collapses, lead to the occurrence of a denser porous crystalline phase of $\mathrm{Zn}(\mathrm{mim})_{2}$, with reduced pore volume compared to ZIF-8. This can be attributed to the limitations of the force field used: by its bonded nature, it does not allow reconstructive transitions to take place in the system. DFT-based molecular dynamics simulations would allow a direct simulation of the amorphization process, but the computational cost of such simulations on a large-scale supercell of ZIF-8 is not within our reach.

In conclusion, we have determined the mechanism of the pressure-induced amorphization of ZIF-8 as a pressure-induced mechanical instability due to shear mode softening. The shear modulus $\left(C_{44}\right)$ of the ZIF-8 cubic framework, which was shown by earlier work to be quite low, actually decreases under compression of the framework. We find that temperature changes do not induce instability of the framework, consistently with the absence of temperature-induced amorphization of ZIF-8. We show that 
the presence of guest molecules in the highly porous ZIF-8 greatly increases its resistance to shear, requiring a higher pressure to amorphize. In essence, we highlight how constant-stress molecular dynamics can be used to investigate pressure- and temperature-induced amorphization and structural transitions.

\section{ASSOCIATED CONTENT}

Supporting Information. Molecular simulation details, values of elastic constants, methane adsorption

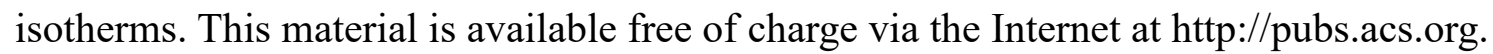

\section{AUTHOR INFORMATION}

\section{Corresponding Author}

*fx.coudert@chimie-paristech.fr

\section{ACKNOWLEDGMENT}

Funding from the Agence Nationale de la Recherche under project "SOFT-CRYSTAB" (ANR-2010BLAN-0822) is acknowledged. This work was performed using HPC resources from GENCI-IDRIS (projects 100254 and 100167), the NAMD software, ${ }^{35}$ which was developed by the Theoretical and Computational Biophysics Group in the Beckman Institute for Advanced Science and Technology at the University of Illinois at Urbana-Champaign.

\section{REFERENCES}

${ }^{1}$ Horike, S.; Shimomura, S.; Kitagawa, S. Soft Porous Crystals. Nat. Chem. 2009, 1, 695-704.

${ }^{2}$ Farha, O. K.; Hupp, J. T. Rational Design, Synthesis, Purification and Activation of Metal-Organic Framework Materials. Acc. Chem. Res. 2010, 43, 1166-1175.

${ }^{3}$ Férey, G. Hybrid Porous Solids: Past, Present, Future. Chem. Soc. Rev. 2008, 37, 191-214. 
${ }^{4}$ Tranchemontagne, D. J.; Mendoza-Cortes, J. L.; O’Keeffe, M.; Yaghi, O. M. Secondary Building Units, Nets and Bonding in the Chemistry of Metal-Organic Frameworks. Chem. Soc. Rev. 2009, 38, $1257-1283$.

${ }^{5}$ Park, K.S.; Ni, Z.; Côté, A.P.; Choi, J.Y.; Huang, R.; Uribe-Romo, F.J.; Chae, H.K.; O’Keeffe, M.; Yaghi, O.M. Exceptional Chemical And Thermal Stability Of Zeolitic Imidazolate Frameworks. Proc. Natl. Acad. Sci. U.S.A. 2006, 103, 10186.

${ }^{6}$ Zhang, J.-P.; Zhu, A.-X.; Lin, R.-B.; Qi, X.-L.; Chen, X.-M. Pore Surface Tailored SOD-Type MetalOrganic Zeolites. Adv. Mater. 2011, 23, 1268-1271.

${ }^{7}$ Phan, A.; Doonan, C. J.; Uribe-Romo, F. J.; Knobler, C. B.; O’Keeffe, M.; Yaghi, O. M. Synthesis, Structure, And Carbon Dioxide Capture Properties Of Zeolitic Imidazolate Frameworks. Acc. Chem. Res. 2010, 43, 58-67.

8 Zhang, J.-P.; Zhang, Y.-B.; Lin, J.-B.; Chen, X.-M. Metal Azolate Frameworks: From Crystal Engineering To Functional Materials. Chem. Rev. 2011, 112, 1001-1033.

${ }^{9}$ Lewis, D.W.; Ruiz-Salvador, A.R.; Gomez, A.; Rodriguez-Albelo, L.M.; Coudert, F.-X.; Slater, B.; Cheetham, A.K.; Mellot-Draznieks, C. Zeolitic Imidazole Frameworks: Structural And Energetics Trends Compared With Their Zeolite Analogues. CrystEngComm 2009, 11, 2272-2276.

${ }^{10}$ Baburin, I.A.; Leoni, S.; Seifert, G. Enumeration Of Not-Yet-Synthesized Zeolitic Zinc Imidazolate Mof Networks: A Topological And Dft Approach. J. Phys. Chem. B 2008, 112, 9437-9443.

11 Hughes, J.T.; Bennett, T.D.; Cheetham, A.K.; Navrotsky, A. Thermochemistry of Zeolitic Imidazolate Frameworks of Varying Porosity. J. Am. Chem. Soc. 2013, 135, 598-601.

12 Li, K.; Olson, D. H.; Seidel, J.; Emge, T. J.; Gong, H.; Zeng, H.; Li, J. Zeolitic Imidazolate Frameworks For Kinetic Separation Of Propane And Propene. J. Am. Chem. Soc. 2009, 131, 1036810369. 
${ }^{13}$ Gücüyener, C.; van den Bergh, J.; Gascon, J.; Kapteijn, F. Ethane/Ethene Separation Turned on Its Head: Selective Ethane Adsorption on the Metal-Organic Framework ZIF-7 through a Gate-Opening Mechanism. J. Am. Chem. Soc. 2010, 132, 17704-17706.

${ }^{14}$ Bux, H.; Feldhoff, A.; Cravillon, J.; Wiebcke, M.; Li, Y-S.; Caro, J. Oriented Zeolitic Imidazolate Framework-8 Membrane with Sharp $\mathrm{H}_{2} / \mathrm{C}_{3} \mathrm{H}_{8}$ Molecular Sieve Separation. Chem. Mater. 2011, 23, 2262-2269.

15 Bae, T.-H.; Lee, J. S.; Qiu, W.; Koros, W. J.; Jones, C. W.; Nair, S. A High-Performance GasSeparation Membrane Containing Submicrometer-Sized Metal-Organic Framework Crystals. Angew. Chem., Int. Ed. 2010, 49, 9863-9866.

${ }^{16}$ Banerjee, R.; Phan, A.; Wang, B.; Knobler, C.; Furukawa, H.; O’Keeffe, M.; Yaghi, O. M. HighThroughput Synthesis of Zeolitic Imidazolate Frameworks and Application to $\mathrm{CO}_{2}$ Capture. Science 2008, 319, 939-943.

${ }^{17}$ Lu, G.; Hupp, J. T. Metal-Organic Frameworks As Sensors: A ZIF-8 Based Fabry-Pérot Device As A Selective Sensor For Chemical Vapors And Gases. J. Am. Chem. Soc. 2010, 132, 7832-7833.

${ }^{18}$ Liedana, N.; Galve, A.; Rubio, C.; Tellez, C.; Coronas, J. CAF@ZIF-8: One-Step Encapsulation Of Caffeine In MOF. ACS Appl. Mater. Interfaces 2012, 4, 5016-5021.

19 Ortiz, A.U.; Boutin, A.; Fuchs, A.H.; Coudert, F.-X. Anisotropic Elastic Properties of Flexible Metal-Organic Frameworks: How Soft are Soft Porous Crystals? Phys. Rev. Lett. 2012, 109, 195502.

${ }^{20}$ Ortiz, A.U.; Boutin, A.; Fuchs, A.H.; Coudert, F.-X. Metal-Organic Frameworks With Wine-Rack Motif: What Determines Their Flexibility And Elastic Properties? J. Chem. Phys. 2013, 138, 174703.

${ }^{21}$ De Toni, M.; Jonchiere, R.; Pullumbi, P.; Coudert, F.-X.; Fuchs, A.H. How Can a Hydrophobic MOF be Water-Unstable? Insight into the Hydration Mechanism of IRMOFs. ChemPhysChem 2012, 13, 3497-3503. 
${ }^{22}$ Tan, J.-C.; Civalleri, B.; Lin, C.-C.; Valenzano, L.; Galvelis, R.; Chen, P.-F.; Bennett, T.D.; Mellot-

Draznieks, C.; Zicovich-Wilson, C.M.; Cheetham, A.K. Exceptionally Low Shear Modulus in a Prototypical Imidazole-Based Metal-Organic Framework. Phys. Rev. Lett. 2012, 108, 095502.

${ }^{23}$ Bennett, T.D.; Goodwin, A.L.; Dove, M.T.; Keen, D.A.; Tucker, M.G.; Barney, E.R.; Soper, A.K.; Bithell, E.G.; Tan, J.-C.; Cheetham, A.K. Structure and Properties of an Amorphous Metal-Organic Framework. Phys. Rev. Lett. 2010, 104, 115503.

24 Bennett, T.D.; Keen, D.A.; Tan, J.-C.; Barney, E.R.; Goodwin, A.L.; Cheetham, A.K. Thermal Amorphization of Zeolitic Imidazolate Frameworks. Angew. Chem. Int. Ed. 2011, 50, 3067-3071.

25 Chapman, K.W.; Halder, G.J.; Chupas, P.J. Pressure-Induced Amorphization and Porosity Modification in a Metal-Organic Framework. J. Am. Chem. Soc. 2009, 131,17546-17547.

26 Cao, S.; Bennett, T.D.; Keen, D.A.; Goodwin, A.L.; Cheetham, A.K. Amorphization Of The Prototypical Zeolitic Imidazolate Framework ZIF-8 By Ball-Milling. Chem. Commun. 2012, 48, 78057807.

27 Bennett, T.D.; Simoncic, P.; Moggach, S.A.; Gozzo, F.; Macchi, P.; Keen, D.A.; Tan, J.-C.; Cheetham, A.K. Reversible Pressure-Induced Amorphization Of A Zeolitic Imidazolate Framework (ZIF-4). Chem. Commun. 2011, 47, 7983-7985.

${ }^{28}$ Ray, J.R. Fluctuations And Thermodynamic Properties Of Anisotropic Solids. J. Appl. Phys. 1982, $53,6441$.

${ }^{29}$ Parrinello, M.; Rahman, A. Strain Fluctuations And Elastic Constants. J. Chem. Phys. 1982, 76, $2662-2666$.

30 Zhang, L.; Hu, Z.; Jiang, J. Sorption-Induced Structural Transition of Zeolitic Imidazolate Framework-8: A Hybrid Molecular Simulation Study. J. Am. Chem. Soc. 2013, 135, 3722-3728.

${ }^{31}$ Morris Jr, J.W.; Krenn, C.R. The Internal Stability Of An Elastic Solid. Philos. Mag. A 2000, 12, 2827-2840. 
${ }^{32}$ Karki, B.B.; Ackland, G.J.; Crain, J. Elastic Instabilities In Crystals From Ab Initio Stress-Strain Relations. J. Phys. Cond. Mat. 1997, 9, 8579-8589.

33 Dean, D.W.; Wentzcovitch, R.M.; Keskar, N.; Chelikowsky, J.R. Pressure-Induced Amorphization In Crystalline Silica: Soft Phonon Modes And Shear Instabilities In Coesite. Phys. Rev. B 2000, 61, 3303-3309.

34 Triguero, C.; Coudert, F.-X.; Boutin, A.; Fuchs, A.H.; Neimark, A.V. Understanding AdsorptionInduced Structural Transitions In Metal-Organic Frameworks: From The Unit Cell To The Crystal. J. Chem. Phys. 2012, 137, 184702.

35 Phillips, J.C.; Braun, R.; Wang, W.; Gumbart, J.; Tajkhorshid, E.; Villa, E.; Chipot, C.; Skeel, R.D.; Kale, L.; Schulten, K. Scalable Molecular Dynamics with NAMD. J. Comput. Chem. 2005, 26, 17811802. 